\title{
The learning curve for transoral incisionless fundoplication
}

\section{다 $(9)$}

\section{Authors}

Mohamad Dbouk*, 1,2, Olaya I. Brewer Gutierrez ${ }^{*}, 1$, Bijun Sai Kannadath³ , Jose Valentin Camilion ${ }^{4}$, Saowanee Ngamruengphong1 ${ }^{1}$, Vivek Kumbhari ${ }^{1}$, Mouen Khashab ${ }^{1}$, Michael Murray ${ }^{5}$, Peter Janu ${ }^{6}$, Glenn Ihde ${ }^{7}, K^{2} n n e t h$ Chang $^{8}$, Nirav Thosani ${ }^{* *}, 9$, Marcia Irene Canto ${ }^{* *}, 1$

Institutions

1 Division of Gastroenterology and Hepatology, Johns Hopkins University School of Medicine, Baltimore, Maryland, United States

2 Department of Pathology, Johns Hopkins University School of Medicine, Baltimore, Maryland, United States

3 Department of Internal Medicine, University of Arizona College of Medicine - Phoenix, Arizona, United States

4 Nova Southeastern College of Osteopathic Medicine, Davie, Florida, USA

5 UNRMed-University of Nevada, Reno, Nevada, United States

6 Fox Valley Surgical Associates, ThedaCare Regional Medical System, Appleton, Wisconsin, United States

7 Matagorda Regional Medical Center, Bay City, Texas, United States

8 H.H. Chao Comprehensive Digestive Disease Center, University of California, Irvine, California, United States

9 Division of Gastroenterology, Hepatology \& Nutrition, McGovern Medical School, UTHealth, Houston, Texas, United States

submitted 5.2.2021

accepted after revision 22.6.2021

\section{Bibliography}

Endosc Int Open 2021; 09: E1785-E1791

DOI 10.1055/a-1547-6599

ISSN 2364-3722

(C) 2021. The Author(s).

This is an open access article published by Thieme under the terms of the Creative Commons Attribution-NonDerivative-NonCommercial License, permitting copying and reproduction so long as the original work is given appropriate credit. Contents may not be used for commercial purposes, or adapted, remixed, transformed or built upon. (https://creativecommons.org/licenses/by-nc-nd/4.0/)

Georg Thieme Verlag KG, Rüdigerstraße 14,

70469 Stuttgart, Germany

Corresponding author

Marcia Irene Canto, Johns Hopkins University - Division of Gastroenterology and Hepatology, Baltimore, Maryland

\author{
21287, United States \\ Fax: +1-410-614-2490 \\ Mcanto1@jh.edu
}

\section{ABSTRACT}

Background and study aims Transoral incisionless fundoplication (TIF) is a safe and effective minimally invasive endoscopic technique for treating gastroesophageal reflux disease (GERD). The learning curve for this technique has not been reported. We studied the learning curve for TIF when performed by a gastroenterologist by identifying the threshold number of procedures needed to achieve consistent technical success or proficiency (consistent creation of TIF valve $\geq 270$ degrees in circumference, $\geq 2 \mathrm{~cm}$ long) and efficiency after didactic, hands-on and case observation experience.

Patients and methods We analyzed prospectively collected data from patients who had TIF performed by a single therapeutic endoscopist within 17 months after basic training. We determined thresholds for procedural learning using cumulative sum of means (CUSUM) analysis to detect changes in achievement rates over time. We used breakpoint analysis to calculate procedure metrics related to proficiency and efficiency.

Results A total of 69 patients had 72 TIFs. The most common indications were refractory GERD (44.7\%) and proton pump inhbitor intolerance (23.6\%). Proficiency was achieved at the $18^{\text {th }}$ to $20^{\text {th }}$ procedure. The maximum efficiency for performing a plication was achieved after the $26^{\text {th }}$ procedure, when mean time per plication decreased to 2.7 from 5.1 minutes $(P<0.0001)$. TIF procedures time varied until the $44^{\text {th }}$ procedure, after which it decreased significantly from 53.7 minutes to 39.4 minutes $(P<0.0001)$. Conclusions TIF can be safely, successfully, and efficiently performed in the endoscopy suite by a therapeutic endoscopist. The TIF learning curve is steep but proficiency can be achieved after a basic training experience and 18 to 20 independently performed procedures.

\footnotetext{
* Drs. Dbouk and Gutierrez contributed equally.
}

** Drs. Thosani and Canto are co-senior authors. 


\section{Introduction}

The most effective and durable anti-reflux intervention is a laparoscopic Nissen fundoplication (LNF) [1-3]. However, alternative treatments for gastroesophageal reflux disease (GERD) have emerged over the last 15 years, including transoral incisionless fundoplication (TIF). It is a safe and effective minimally invasive endoscopic technique for the management of GERD in selected patients with small hiatal hernias $\leq 2 \mathrm{~cm}$ and small diaphragmatic defects (Hill grade 1-2) [4-7]. Introduced in 2005, TIF is an endoscopic procedure that creates a flap valve with full-thickness serosa-serosa plications of the esophagus and gastric cardia, with the aim of restoring the angle of His, similar to a surgical partial fundoplication $[6,8]$. Multiple randomized controlled clinical trials report resolution of troublesome regurgitation not responding to proton pump inhibitor (PPI) therapy in the majority of patients treated with TIF [4,5,9-12]. In particular, a randomized controlled trial published in 2015 by Trad et al (the TEMPO trial) showed that TIF was more effective than PPI therapy for refractory GERD symptoms [5]. The followup study reported durable symptom resolution of $88 \%$ at 1 year, $90 \%$ at 3 years, and $88 \%$ at 5 years [13]. Furthermore, a systematic review and meta-analysis performed by McCarty et al of 32 studies (involving 1475 patients) concluded that TIF is a technically feasible and effective endoscopic procedure for the management of GERD with a technical success rate of $99 \%$ and low rate $(2 \%)$ of serious adverse effects [11]. Another systematic review with network meta-analysis showed that TIF is associated with a higher likelihood of improved health-related quality of life compared to PPI and laparoscopic Nissen fundoplication [14].

In the published clinical trials, experienced surgeons and gastroenterologists performed TIF $[4,5,12,13,15,16]$. There are very limited data on the learning curve of TIF when performed by a gastroenterologist. We present a single gastroenterologist's early experience to study the learning curve of TIF and propose threshold procedure volumes to achieve proficiency and efficiency.

\section{Patients and methods}

This was an observational study conducted in a single American tertiary care academic center. Data were collected prospectively on consecutive patients referred to the Johns Hopkins Heartburn Center who had a TIF procedure performed by an experienced therapeutic endoscopist (MC) between September 2017 and February 2020. All patients provided written informed consent to participate in the Johns Hopkins Heartburn Center (TIF) Registry, which prospectively tracks patients undergoing TIF at the Johns Hopkins Hospital (Johns Hopkins IRB number IRB00201157; clinicaltrials.gov NCT03853772). Prior to performing TIF without supervision, the gastroenterologist acquired cognitive knowledge and technical training on performance of TIF by review of a standardized curriculum in a classroom-type setting, hands-on performance of TIF on an inanimate and live animal model, and in-person observation of cases performed by a highly experienced gastroenterologist who achieved expertise in TIF (KC). These observed were not included in the TIF learning curve.

Currently, TIF using the 2.0 technique is performed with the EsophyX-Z or Esophyx-Z+ device (EndoGastric Solutions, Redmond, WA) under general anesthesia with naso- or endotracheal intubation ( $\triangleright$ Fig.1) [17]. The EsophyX device with videoendoscope inserted is advanced into the stomach, and endoscopic fundoplication is achieved in the retroflex position. Small hiatal hernias can be reduced by using a helical retractor that is inserted into the gastroesophageal junction and gently pulled down the esophagus below the diaphragm while decompressing the stomach. The fundoplication is created through clockwise and counterclockwise wrapping of tissue at the gastric cardia at the 11 o'clock and 1 o'clock positions and placement of multiple transmural esophago-gastric plications using polypropylene $\mathrm{H}$-fasteners with comparable strength to $3.0 \mathrm{su}$ tures placed to create a fundoplication that is at least 270 degrees in circumference [17]. Valve length is achieved by withdrawing the esophagus from 2 to $4 \mathrm{~cm}$ into the stomach and placing fasteners at intervals between the 11 and 1 o'clock positions.

Routine pre-TIF work-up included upper endoscopy (EGD) with biopsy and careful evaluation of esophageal landmarks, cine-esophagogram, high-resolution esophageal manometry (HREM) (in patients with dysphagia) and ambulatory $\mathrm{pH}$-monitoring with either 4-day Bravo wireless $\mathrm{pH}$ capsule off medications or 24-hour pH impedance. Patients were considered appropriate for TIF if they had an abnormal $\mathrm{pH}$-test (acid exposure time $>6 \%$ ), hiatal hernia $\leq 2 \mathrm{~cm}$, Hill grade $\leq 2$, and a $\mathrm{BMI}<35$ [5]. Patients who had per-oral endoscopic myotomy (POEM) prior to TIF were excluded from the analysis. TIF was performed using the EsophyXZ or EsophyX Z + device and the TIF 2.0 protocol [17].

Patient demographic information, axial length of any hiatal hernia, Hill classification of the gastroesophageal flap valve [18], TIF procedure indication, location and number of plications, total number of fasteners placed, TIF valve circumferential extent, TIF valve length, and TIF procedure time (time from first passage to withdrawal of the EsophyX device) were recorded on a secure online research electronic data capture system (REDCap software, Vanderbilt University, Nashville, Tennessee, United States). Two independent observers who were not part of the physician or nursing team assessed the final TIF valve length and circumference.

\section{Statistical analysis}

The primary endpoint of the study was the threshold number of procedures needed to achieve proficiency (defined as creation of a TIF valve $\geq 270$ degrees in circumference and $>2 \mathrm{~cm}$ in length) in performing TIF. The secondary endpoints were the threshold number of TIF procedures to achieve efficiency (defined by the minimum mean time to perform a TIF) and the minimum mean time to perform a plication (place a set of two fasteners).

Patients and disease characteristics were described as frequencies with percentages (\%), medians [IQR], and means \pm standard deviation (SD). We used the Cumulative Sum of 


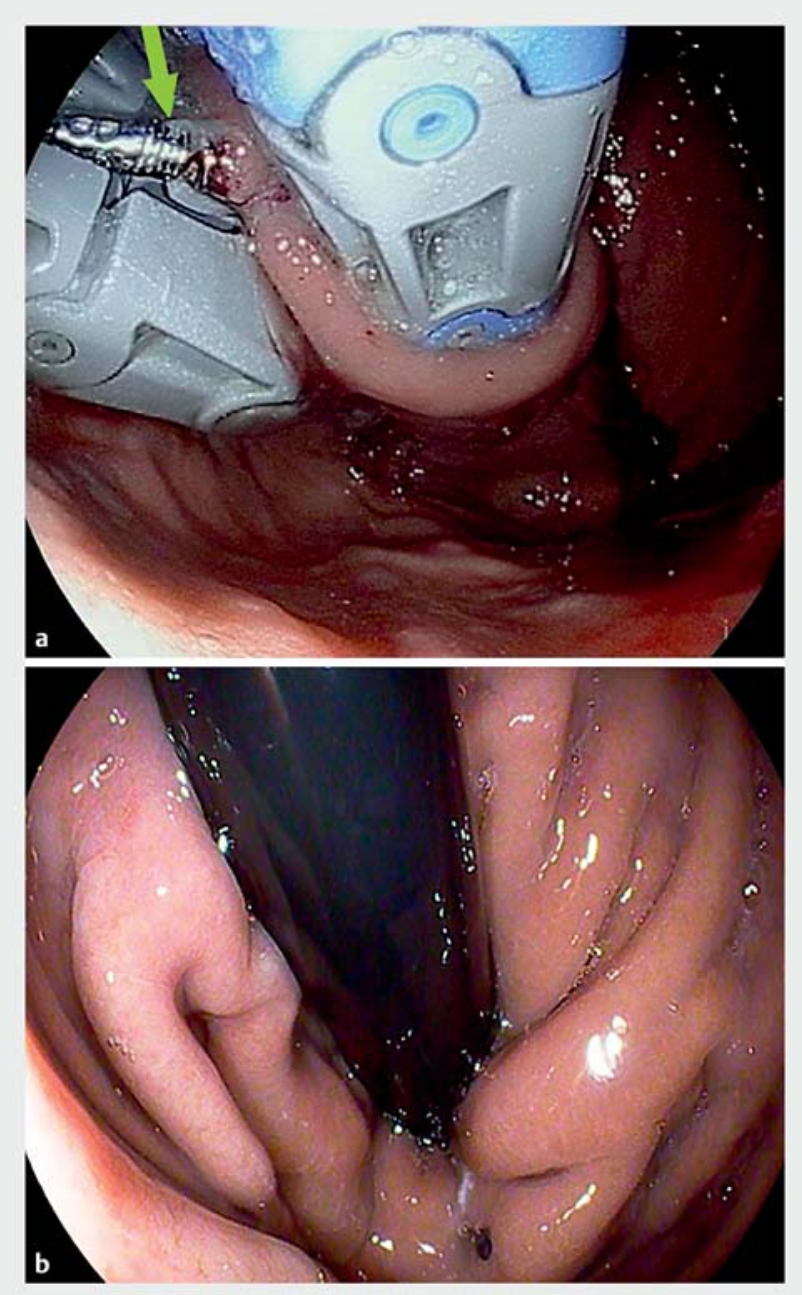

- Fig. 1 Endoscopic images of transoral incisionless fundoplication using TIF 2.0 technique. a Endoscopic image of the Esophyx-Z+ device seen in retroflex view during the initial step of transoral incisionless fundoplication. The helix (arrow) is inserted into the gastric cardia side of the squamocolumnar junction, and with concomitant suctioning, the distal esophagus is withdrawn in caudad direction under the diaphragm to create a flap valve 2 to 3 $\mathrm{cm}$ in length. The distal esophagus and gastric cardia are shown enclosed within the tissue mold prior to placement of plastic $\mathrm{H}$ fasteners to create a full thickness esophago-gastric plication. In TIF 2.0 technique, at least 20 fasteners are placed at different locations to create a flap valve that is at least 270 degrees in length. b The endoscopic image on the right is of the TIF valve 6 months later and shows the gastric cardia snug around the endoscope (normal appearance, Hill grade 1), with a 310-degree circumference.

Means (CUSUM) analysis to estimate the threshold for achieving proficiency by detecting sequential changes in achievement rates over time, as the endoscopist gained experience from each procedure. The essential function of CUSUM is to calculate the trends in "average success rates" over time. We calculated the average success rate at the completion of each procedure, and plotted the changes in this rate. In order to detect changes occuring in various procedure parameters and metrics as the operators experience with the procedure increased, we checked for structural breaks in the time series data, i.e. we looked for breakpoints around which the means varied greatly before and after. Specifically, we determined the number of procedures needed to minimize total TIF procedure time, time to perform a plication (in minutes), and to consistently achieve a $\geq 270$ circumferential degree of wrap. We performed data analyses and generated figures using Stata 15 (StataCorp, College Station, Texas, United States). All authors had access to the study data and reviewed and approved the final manuscript.

\section{Results}

A total of 69 patients (63.8\% were males with a mean age of 54 \pm 13 years) had 72 TIFs after work-up confirmed appropriate criteria (abnormal pH-metry, $<2 \mathrm{~cm}$ hiatal hernia $<2 \mathrm{~cm}$, Hill grade $\leq 2$, BMI $<35)$. Six patients $(8.3 \%)$ had a history of prior surgical fundoplication. The primary indications for TIF were refractory GERD (44.7\%), PPI-averse patients (23.6\%), postablation of Barrett's esophagus (11.1\%), laryngopharyngeal reflux disease (LPRD) (11.1\%), and failed Nissen fundoplication (8.3\%). The median hiatal hernia axial length was 1 [IQR 01.37]; the majority of patients had no hiatal hernia $(37.5 \%)$ or a hiatal hernia measuring $1 \mathrm{~cm}$ (33.3\%). The median Hill grade was 1 [IQR 1-2] ( Table 1).

The overall procedure completion rate was $98.5 \%$. One TIF procedure (TIF \#7) was not completed due to inability to reduce the hiatal hernia. Subsequent laparoscopic anti-reflux surgery showed a $2-3 \mathrm{~cm}$ hiatal hernia and large crural defect with fat pad, likely causing underestimation of the hiatal hernia length and width.

All but one patient (admitted for 23 hours for a migraine headache, unrelated to the TIF procedure) were discharged home. There were no procedure-related serious adverse events. The mean TIF valve circumference was $290 \pm 22.3$ degrees. The mean TIF procedure time was $48.1 \pm 13.9$ minutes.

\section{Outcomes}

Overall, $89 \%$ of all procedures led to the creation of a TIF valve with a minimum circumference of 270 degrees and minimum length $>2 \mathrm{~cm}$. Notably, three TIFs completed and performed (TIF \# 3,8,15) during the learning phase did not meet the technical success criteria. In retrospect, these patients met criteria for TIF at a prior EGD but at the TIF procedure had larger hiatal hernia and/or Hill grade when prior images were reviewed ( $\triangleright$ Table 1). In addition, three patients who had their TIF in the early part of the learning curve successfully underwent repeat TIF later on due to symptom recurrence. CUSUM analysis of achievement of technical success revealed an inverted $V$ pattern, indicating acquired proficiency from the 20th procedure $(P=0.005)$ ( Fig. 2). Review of the primary data showed that all TIF procedures were successful and met target TIF valve parameters from the $18^{\text {th }}$ TIF procedure.

Breakpoint analysis revealed that the threshold to achieve proficiency was achieved at the 20th procedure $(P<0.0001)$ ( $\triangleright$ Fig.3), consistent with the CUSUM analysis. Review of the primary procedure data showed that all TIF valves exceeded the minimum threshold circumference of 270 degrees from 


\begin{tabular}{|c|c|}
\hline Patients & $N=69$ \\
\hline Mean age, SD & $54($ SD 13) \\
\hline Gender, male & $44(63.8 \%)$ \\
\hline Mean BMI, SD & $27.3($ SD 6.3$)$ \\
\hline History of Barrett's esophagus & $16(23.2 \%)$ \\
\hline Esophagitis pre-TIF & $17(24.6 \%)$ \\
\hline LA Grade A & $8(11.6 \%)$ \\
\hline LA Grade B & $5(7.2 \%)$ \\
\hline LA Grade C & $4(5.8 \%)$ \\
\hline Abnormal average acid exposure time (AET) ${ }^{1}$ & $31(44.9 \%)$ \\
\hline Average acid exposure time (AET), median (IQR) & $6.5(3.7-8.7)$ \\
\hline Primary Indication for TIF & \\
\hline Refractory GERD & $30(41.7 \%)$ \\
\hline LPRD & $8(11.1 \%)$ \\
\hline Failed Nissen & $6(8.3 \%)$ \\
\hline Failed TIF & $3(4.2 \%)$ \\
\hline PPI-averse & $17(23.6 \%)$ \\
\hline Post-ablation BE & $8(11.1 \%)$ \\
\hline Procedures & $(n=72)$ \\
\hline $\begin{array}{l}\text { Median hiatal hernia axial length }(\mathrm{cm}) \text { (at the TIF } \\
\text { procedure) }\end{array}$ & 1 (IQR 0-1.37) \\
\hline Hiatal hernia $=0$ & $28(38.9 \%)$ \\
\hline Hiatal hernia $=1 \mathrm{~cm}$ & $25(34.7 \%)$ \\
\hline Hiatal hernia between 1 to $2 \mathrm{~cm}$ & $2(2.7 \%)$ \\
\hline Hiatal hernia $=2 \mathrm{~cm}$ & $17(23.6 \%)$ \\
\hline Median Hill grade (at the TIF procedure) & $1(\mathrm{IQR} 1-2)$ \\
\hline Hill grade 1 & $42(58.3 \%)$ \\
\hline Hill grade 2 & $27(37.5 \%)$ \\
\hline Hill grade 3 & $3(4.1 \%)$ \\
\hline \multicolumn{2}{|c|}{$\begin{array}{l}\text { SD, standard deviation; BMI, body mass index; IQR, interquartile range; AET, } \\
\text { abnormal average acid exposure time; TIF, transoral incisionless fundoplica- } \\
\text { tion; GERD, gastroesophageal reflux disease; LPRD, laryngopharyngeal re- } \\
\text { flux disease; PPI, proton pump inhibitor; BE, Barrett's esophagus. } \\
{ }^{1} \text { AET > } \% \text { off acid suppressive medication. }\end{array}$} \\
\hline
\end{tabular}

the $18^{\text {th }}$ procedure. With regards to achievement of efficiency, the threshold to improve the time for each plication was achieved at the 26 th procedure $(P<0.0001)$ ( $>$ Fig. 4$)$. The overall mean time to perform a plication was $3.65 \pm 1.56$ minutes, which significantly decreased after achieving proficiency (mean time $=2.8 \mathrm{~min} \pm 0.7$ per plication $(95 \% \mathrm{Cl} 2.6-3 \mathrm{~min}$ utes), compared to $5.1 \pm 1.8$ ( $95 \% \mathrm{Cl} 4.39-5.81$ minutes) before $26^{\text {th }}$ procedure, $(P<0.0001)$. Finally, the threshold to improve the TIF procedure time was noted at the 44th procedure ( $\triangleright$ Fig.5). The average TIF procedure time gradually decreased

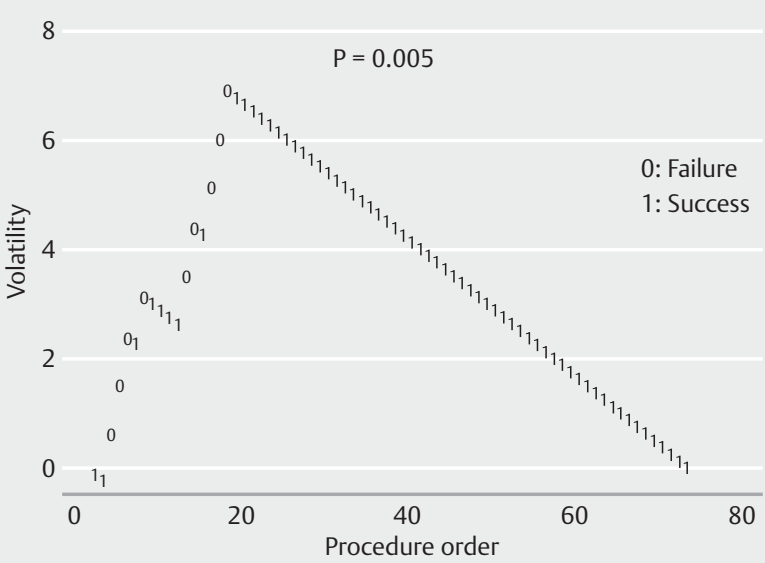

Fig. 2 Cumulative sum (CUSUM) analysis to assess procedure proficiency. (consistent creation of a $\geq 270$-degree and $>2$-cmlong TIF valve).

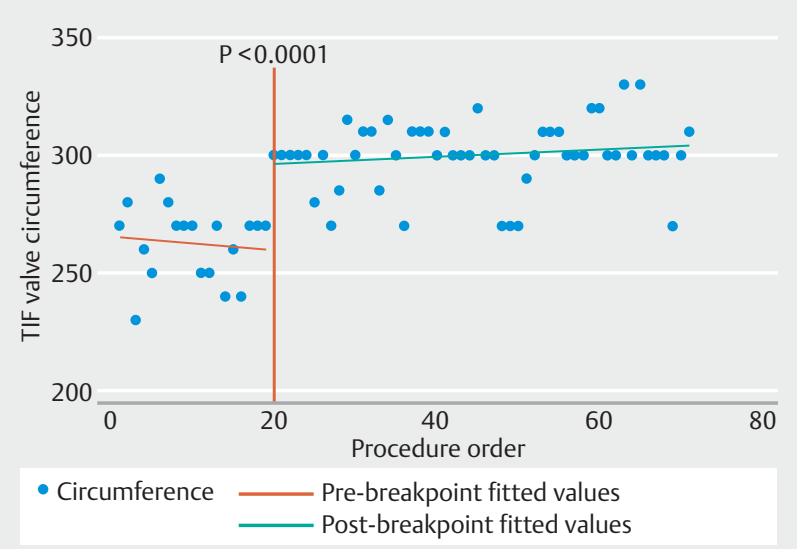

- Fig. 3 Breakpoint analysis of TIF valve circumference. (Proficiency: consistent creation of a minimum 270-degree TIF wrap).

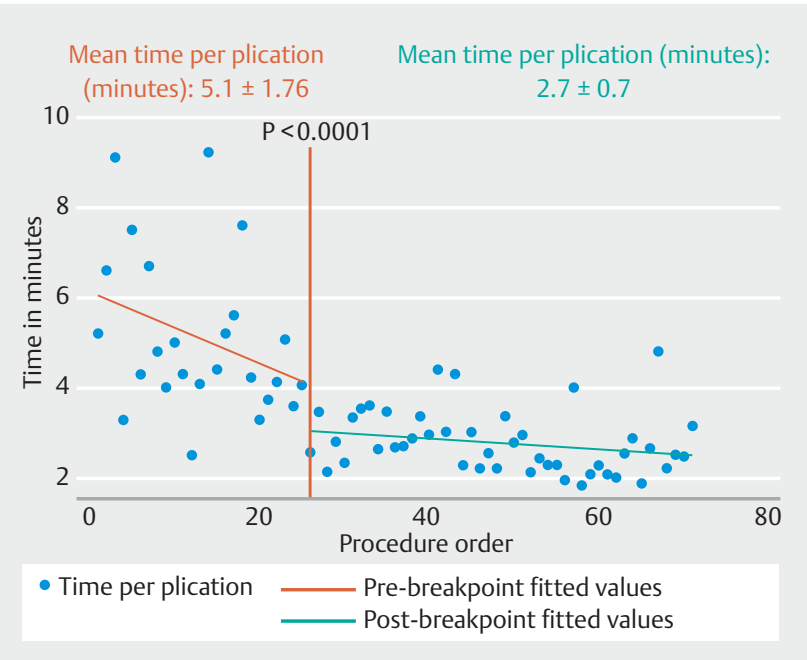

- Fig. 4 Breakpoint analysis of average plication time. 


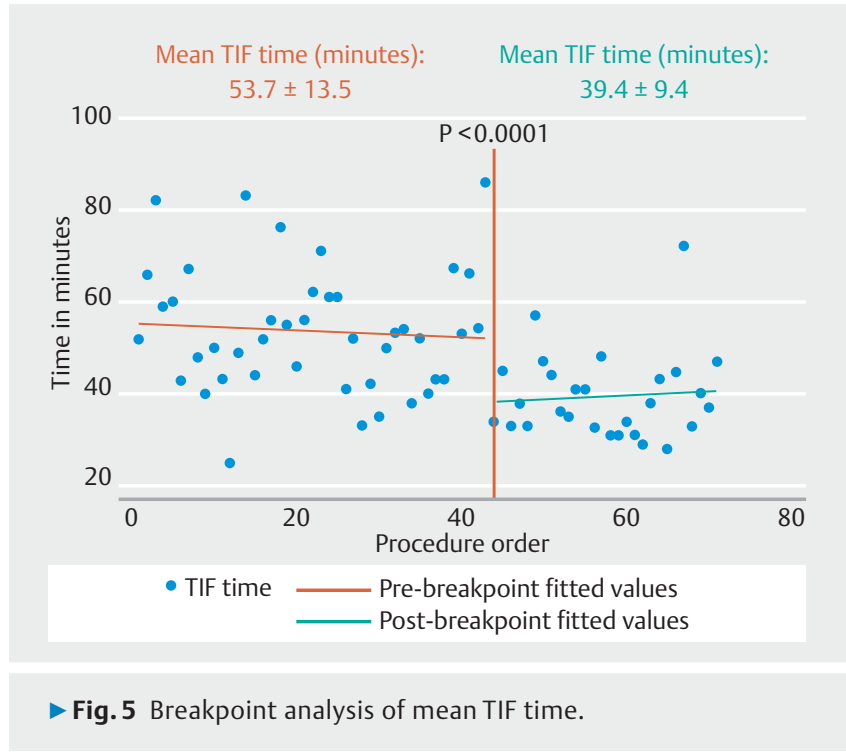

with increasing experience, but after the $44^{\text {th }}$ procedure the mean TIF time decreased significantly to 39.4 minutes $(95 \% \mathrm{CI}$ 9.4), compared to 53.7 minutes ( $95 \% \mathrm{Cl} 13.5)$ before the breakpoint $(P<0.0001)$.

\section{Discussion}

In this single-center study, we report the early experience of a therapeutic endoscopist and described for the first time the learning curve for TIF performed entirely in the endoscopy unit on an outpatient basis. We evaluated several metrics for estimating thresholds for achieving proficiency and efficiency. All of the metrics revealed two distinct phases in the learning curve: an early phase and a later phase. The breakpoints for achieving proficiency and efficiency were different. Proficiency was achieved after 18 to 20 TIF procedures, efficiency for performing plications was achieved after 26 procedures, and maximum efficiency (reduced TIF time) was achieved after 44 procedures, suggesting differences in achieving proficiency in various aspects of the procedure.

To our knowledge, this is the first report of the TIF learning curve. In a preliminary report, Raza et al suggested that TIF could be safely performed by trained therapeutic endoscopists in the endoscopy suite with no intra-procedure or post-procedure adverse events [17]. Also, it showed that all patients could safely be discharged within 24 hours post-procedure [19]. Similarly, our study suggests that TIF can be performed safely and successfully by an experienced gastroenterologist in the endoscopy unit after a standardized didactic and observational experience.

Other investigators have investigated the learning curves of complex endoscopic procedures using different approaches, often including decrease in procedure time. For example, a single-center retrospective study performed by El Zein et al described the learning curve for POEM by identifying a threshold number of procedures required to significantly lower procedure time. With a single experienced therapeutic endoscopist per- forming all the procedures, the investigators concluded that a threshold number of 13 POEM procedures will lead to a learning plateau [20]. Similarly, the learning curve of a single therapeutic endoscopist for endoscopic sleeve gastroplasty (ESG) using procedure time and number of plications per procedure showed that these parameters plateau at seven and nine cases, respectively [21]. Both single operator studies suggest a very short, steep learning curve for complex endoscopic procedures if time is the main parameter to assess learning. In contrast, the learning curve for therapeutic endoscopic procedures can also be assessed using defined proficiency benchmarks as well as procedure time. For example, in a large retrospective study of 1346 patients, Liu et al described the learning curve of POEM using both procedure time and a composite outcome of technical failure, adverse events, and clinical failure, suggesting 100 cases were needed, despite prior experience in endoscopic submucosal dissection (ESD) and a high POEM volume [22].

Other learning curve studies also emphasized achievement of specific goals and not procedure time. Using a porcine exvivo model (Erlangen Active Simulator for Interventional Endoscopy), Gonzalez et al conducted a prospective ex-vivo learning curve study based on the number of EUS-FNA procedures performed on a cyst and two solid masses for assessing learning, such as number of successful attempts, needle view loss, and scope handling $[23,24]$. Zhang et al performed a retrospective analysis of consecutive ESD to describe the learning curve of a single operator between 2009 and 2017 [25]. The authors proposed that to achieve proficiency - defined as $\geq 90 \%$ en block resection and $>80 \%$ margin-negative resection with resection speed of $>9 \mathrm{~cm}^{2}$ per hour -250 cases are needed. Similarly, ERCP procedure threshold for trainees has been proposed, based on success of common bile duct cannulation [26].

In the current study, we used a combination of procedure time and technical success to assess the learning curve for TIF. Using a predefined proficiency benchmark (creation of a TIF 2.0 valve $\geq 270$ degrees and $>2 \mathrm{~cm}$ long), we demonstrated a steep rise in the learning curve, with proficiency achieved after 18 to 20 procedures. When we tracked proficiency and efficiency using time as the endpoint, we found that with increased experience, efficiency improves after achieving proficiency and the TIF learning curve using time plateaus. It is possible that additional experience would further decrease procedure time and improve efficiency beyond the 72 procedures included in this early experience.

It is important to mention that TIF is not routinely performed at most medical centers. It is also not routinely taught in gastroenterology and surgical training programs. There is a standard set of required reading and ex vivo inanimate model technical training offered to new users. But beyond this, observation or participation in clinical cases and supervised learning may vary across different practice settings. It is important to standardize the learning experience and address potential pathways for training to enhance clinical adoption of this relatively newer anti-reflux procedure that gastroenterologists can offer patients with suitable anatomy.

Finally, our early TIF experience underscores the importance of estimating the length and width of the hiatal hernia. The Hill 
grade (a better estimate of the hiatal hernia width and crural defect) [27] is critical factor in the success of TIF without hiatal hernia repair. TIF can typically reduce small hiatal hernias $\leq 2 \mathrm{~cm}$ in length, but larger and wider hernias require surgical repair and cruroplasty. The hiatal hernia length and width can be underestimated during endoscopy, as shown in our 4 TIF technical failures. Better methods for accurate estimation of the hiatal hernia size, standardization of the best method and reporting of findings, and widespread education of endoscopists on the proper assessment of the anti-reflux barrier are needed.

The strengths of our study include the documentation of the learning experience prior to independent procedure performance, prospective data collection, use of time as well as objective procedure metrics, independent observer assessment of the endpoints (TIF valve characteristics), and quantitative analyses using two statistical approaches demonstrating consistency in our results. However, there are also certain limitations to our study. First, it was a single-center study and reports a single endoscopist's experience. Hence, generalizability is limited. There is certainly going to be some variability in the learning curves and number of TIF procedures needed to achieve technical success among different operators. It would be interesting to study the learning curve of different specialties and experience levels, such as trainees, general surgeons, specialized foregut surgeons, and therapeutic endoscopists. Second, our study included TIF data on a limited number of patients, but our sample size of 72 TIF procedures approaches or exceeds that in some multicenter randomized controlled trials where TIF was performed by more than one operator $[5,6,12,28]$. Finally, we report the learning curve for TIF only considering proficiency and efficiency. Larger multicenter studies including hundreds of procedures that assess the learning of TIF considering all aspects (technical success, safety, and clinical outcomes), similar to the study reported for the learning curve of radiofrequency ablation [29], are needed. These studies are already underway (NCT03853772). Moreover, it would be important to understand prior operator experience and assess its impact on safety and efficacy in future clinical trials involving TIF, because early clinical trials did not routinely document this. Nevertheless, despite these study limitations, this is the first prospective study of the learning curve for TIF 2.0, which provides a basic understanding of procedural experience needed to potentially assist future research studies, training programs, and hospital credentialing committees.

\section{Conclusions}

In conclusion, TIF can be safely, successfully, and efficiently performed in the endoscopy suite by a gastroenterologist. Proficiency can be achieved after 18 to 20 procedures, while efficiency is optimal after the $44^{\text {th }}$ procedure. Future studies should correlate the learning curve with efficacy.

\section{Competing interests}

Dr. Ngamruengphong is a consultant for Boston Scientific. Dr. Kumbhari is a consultant for Medtronic, Pentax Medical (USA), Boston Scientific, FujiFilm, Apollo Endosurgery, and received research support from Erbe USA, and Apollo Endosurgery. Dr. Khashab is a consultant for Boston Scientific, Medtronic, Olympus, GI Supply, and Triton. Dr. Murray is a consultant for Endogastric Solutions. PJ is a consultant for Endogastric solutions, Ethicon/J\&J, and Olympus. Dr. Ihde is a consultant for Endogastric solutions, and Microline Medical. Dr. Chang is a consultant for Apollo Endosurgery, Cook, Erbe USA, Endogastric solutions, Mauna Kea, Mederi, Medtronic, Olympus, Ovesco and Pentax Medical (USA). Dr. Thosani is a consultant for Boston Scientific, Medtronic, Pentax Medical (USA), received research support from Pentax Medical (USA), royalties from UpToDate, and is on the advisory board of ColubrisMX. Dr. Canto received research grants from Endogastric solutions and Pentax Medical (USA), and royalties from UpToDate.

\section{References}

[1] Du X, Wu JM, Hu ZW et al. Laparoscopic Nissen (total) versus anterior $180^{\circ}$ fundoplication for gastro-esophageal reflux disease: A meta-analysis and systematic review. Medicine (Baltimore) 2017; 96: e8085

[2] Moore M, Afaneh C, Benhuri D et al. Gastroesophageal reflux disease: A review of surgical decision making. World J Gastrointest Surg 2016; 8: 77-83

[3] Migaczewski M, Pędziwiatr M, Matłok M et al. Laparoscopic Nissen fundoplication in the treatment of Barrett's esophagus - 10 years of experience. Wideochir Inne Tech Maloinwazyjne 2013; 8: 139-145

[4] Hakansson B, Montgomery M, Cadiere GB et al. Randomised clinical trial: transoral incisionless fundoplication vs. sham intervention to control chronic GERD. Aliment Pharmacol Ther 2015; 42: 1261-1270

[5] Trad KS, Barnes WE, Simoni G et al. Transoral incisionless fundoplication effective in eliminating GERD symptoms in partial responders to proton pump inhibitor therapy at 6 months: the TEMPO Randomized Clinical Trial. Surg Innov 2015; 22: 26-40

[6] Cadiere GB, Buset M, Muls V et al. Antireflux transoral incisionless fundoplication using EsophyX: 12-month results of a prospective multicenter study. World J Surg 2008; 32: 1676-1688

[7] Testoni PA, Testoni S, Mazzoleni G et al. Long-term efficacy of transoral incisionless fundoplication with Esophyx (Tif 2.0) and factors affecting outcomes in GERD patients followed for up to 6 years: a prospective single-center study. Surg Endosc 2015; 29: 2770-2780

[8] Thosani N, Goodman A, Manfredi M et al. Endoscopic anti-reflux devices (with videos). Gastrointest Endosc 2017; 86: 931-948

[9] Gerson L, Stouch B, Lobontiu A. Transoral incisionless fundoplication (TIF 2.0): A meta-analysis of three randomized, controlled clinical trials. Chirurgia 2018; 113: 173-184

[10] Huang X, Chen S, Zhao $\mathrm{H}$ et al. Efficacy of transoral incisionless fundoplication (TIF) for the treatment of GERD: a systematic review with meta-analysis. Surg Endosc 2017; 31: 1032-1044

[11] McCarty TR, Itidiare M, Njei B et al. Efficacy of transoral incisionless fundoplication for refractory gastroesophageal reflux disease: a systematic review and meta-analysis. Endoscopy 2018; 50: 708-725

[12] Muls V, Eckardt AJ, Marchese M et al. Three-year results of a multicenter prospective study of transoral incisionless fundoplication. Surg Innov 2013; 20: 321-330

[13] Trad KS, Barnes WE, Prevou ER et al. The TEMPO trial at 5 Years: transoral fundoplication (TIF 2.0) is safe, durable, and cost-effective. Surg Innov 2018; 25: 149-157

[14] Richter JE, Kumar A, Lipka S et al. Efficacy of laparoscopic nissen fundoplication vs transoral incisionless fundoplication or proton pump 
inhibitors in patients with gastroesophageal reflux disease: a systematic review and network meta-analysis. Gastroenterology 2018; 154: $1298-1308$ e7

[15] Bell RC. Randomized controlled trial of transoral incisionless fundoplication vs. proton pump inhibitors for treatment of gastroesophageal reflux disease. Am J Gastroenterol 2015; 110: 1621-1623

[16] Bell RC, Mavrelis PG, Barnes WE et al. A prospective multicenter registry of patients with chronic gastroesophageal reflux disease receiving transoral incisionless fundoplication. J Am Coll Surg 2012; 215 : 794-809

[17] Bazerbachi F, Krishnan K, Abu Dayyeh BK. Endoscopic GERD therapy: a primer for the transoral incisionless fundoplication procedure. Gastrointest Endosc 2019; 90: 370-383

[18] Hill LD, Kozarek RA. The gastroesophageal flap valve. J Clin Gastroenterol 1999; 28: 194-197

[19] Raza AM, Catalano MM, Rahimi EFM et al. Efficacy and safety of transoral incisionless fundoplication performed by gastroenterologists. Am J Gastroenterol 2018; 113: S698-S700

[20] El Zein M, Kumbhari V, Ngamruengphong S et al. Learning curve for peroral endoscopic myotomy. Endosc Int Open 2016; 4: E577-E582

[21] Hill C, El Zein M, Agnihotri A et al. Endoscopic sleeve gastroplasty: the learning curve. Endosc Int Open 2017; 5: E900-E904

[22] Liu Z, Zhang X, Zhang W et al. Comprehensive evaluation of the learning curve for peroral endoscopic myotomy. Clin Gastroenterol Hepatol 2018; 16: 1420-6 e2
[23] Gonzalez JM, Cohen J, Gromski MA et al. Learning curve for endoscopic ultrasound-guided fine-needle aspiration (EUS-FNA) of pancreatic lesions in a novel ex-vivo simulation model. Endosc Int Open 2016; 4: E1286-E1291

[24] Wani S, Keswani RN, Petersen B et al. Training in EUS and ERCP: standardizing methods to assess competence. Gastrointest Endosc 2018; 87: $1371-1382$

[25] Zhang X, Ly EK, Nithyanand S et al. Learning curve for endoscopic submucosal dissection with an untutored, prevalence-based approach in the United States. Clin Gastroenterol Hepatol 2020; 18: 580-8 e1

[26] Ekkelenkamp VE, Koch AD, Rauws EA et al. Competence development in ERCP: the learning curve of novice trainees. Endoscopy 2014; 46: 949-955

[27] Hansdotter I, Bjor O, Andreasson A et al. Hill classification is superior to the axial length of a hiatal hernia for assessment of the mechanical anti-reflux barrier at the gastroesophageal junction. Endosc Int Open 2016; 4: E311-E317

[28] Witteman BP, Conchillo JM, Rinsma NF et al. Randomized controlled trial of transoral incisionless fundoplication vs. proton pump inhibitors for treatment of gastroesophageal reflux disease. Am J Gastroenterol 2015; 110: 531-542

[29] Pasricha S, Cotton C, Hathorn KE et al. Effects of the learning curve on efficacy of radiofrequency ablation for Barrett's esophagus. Gastroenterology 2015; 149: 890-6 e2 